\title{
An Efficient Analysis of Shielded Single and Multiple Coupled Microstrip Lines With the Nonuniform Fast Fourier Transform (NUFFT) Technique
}

\author{
Ke-Ying Su and Jen-Tsai Kuo, Senior Member, IEEE
}

\begin{abstract}
A nonuniform fast Fourier transform (NUFFT) technique is incorporated into the spectral-domain approach for the analysis of shielded single and multiple coupled microstrip lines. Each of the spectral-domain Green's functions is decomposed into an asymptotic part and a remaining part. At the interface of layered dielectrics with conducting strips, the product of a basis function and an associated Green's function constitutes an expansion $E$-field. The inverse Fourier transform (IFT) of the expansion $E$-field is its spatial distribution all over the interface. We take this advantage to match the final boundary conditions on all the conducting strips simultaneously. As a result, if all the strips are at one interface, the number of operations required in this method is proportional to $N_{\ell}$, but not to $N_{\ell}^{2}$, where $N_{\ell}$ is the number of the strips. The IFT of the asymptotic part of each expansion $E$-field can be obtained analytically, and that of the remaining part can be quickly processed by the NUFFT. The Gauss-Chebyshev quadrature is used to accelerate the computations of the integrals resulted from the Galerkin's procedure. The proposed method is also applied to investigate the dispersion characteristics of coupled lines with finite metallization thickness and of coupled lines at different levels. A convergence analysis of the results is presented and a comparison of used CPU time is discussed.
\end{abstract}

Index Terms-Method of moments (MoM), microstrip lines, nonuniform fast Fourier transform (NUFFT), spectral-domain approach (SDA).

\section{INTRODUCTION}

$\mathbf{T}$ HE ANALYSIS of single and multiple coupled microstrip lines (MCMLs) has attracted attention from many researchers. Numerical methods, like finite difference time domain (FDTD) [1] and mature commercial finite-element simulators such as Ansoft High Frequency Structure Simulator (HFSS), have good flexibility and efficiency in accurately determining the dispersive characteristics and normal-mode parameters of MCMLs. However, analytical formulations are usually preferred when detailed characteristics of dominant higher order, evanescent and complex modes are required. For some tough structures, as it will be reported later, the analysis even requires a solution procedure capable of providing results with a very high resolution. In such cases, an analytical formu-

Manuscript received February 18, 2003; revised August 11, 2003. This work was supported in part by the National Science Council, Taiwan, R.O.C., under Grant NSC 91-2213-E-009-126, and in part by the joint program of the Ministry of Education and the National Science Council under Contract 89-E-F-A06-2-4.

The authors are with the Department of Communication Engineering, National Chiao Tung University, Hsinchu, 300, Taiwan, R.O.C. (e-mail: jtkuo@cc.nctu.edu.tw).

Digital Object Identifier 10.1109/TMTT.2003.821248 lation, like the spectral-domain approach (SDA), is promising to provide correctly converged results [2].

The mixed-potential integral equation (MPIE), in conjunction with the complex image method [3], can perform the evaluation of Galerkin's matrix entries in a very efficient way. The discrete space-domain formulation [4], [5] can be used to calculate a large number of higher order modes. The SDA [2], [6]-[10] are also widely used analytical formulations in analyzing microstrip structures.

Accelerating the SDA has been an important issue in the past decades. This is because when any strip has a narrow width or any dielectric layer has a small thickness, a very large number of spectral terms must be taken into account [5]. It has been shown that more than $10^{4}$ spectral terms are required to calculate the attenuation constant of a microstrip [7] and the propagation constant of a complex mode of tightly coupled microstrips [2].

Many authors employ an asymptotic extraction technique [3]-[5], [8], [9] to deal with the acceleration. In this technique, closed-form asymptotes are extracted from the spectral-domain Green's functions, and the remaining terms are processed separately. In [3], the extracted functions are transformed into the spatial domain, where the matching of the final boundary conditions on conducting strips is also performed. In [4] and [5], the Galerkin's procedure is performed in the spectral domain. For a single microstrip [8] and symmetric coupled microstrips [9] in an open structure, closed forms for the asymptotic parts of the impedance matrix elements are derived.

The remaining terms have an important contribution to the final method of moments (MoM) matrix. In [3], the remaining terms of the Green's function are expanded as a finite sum of complex exponential functions and transformed to the spatial domain. In [4], [5], [8], and [9], the remaining parts of the impedance matrix elements are directly tackled by numerical calculations since the remaining functions decay quickly when the spectral variable is increased.

Recently, a rapidly converging SDA is presented for the exact analysis of shielded layered microstrips [10]. The direct integral-equation technique optimizes the MoM by recasting all matrix elements into rapidly converging series. The presented results, however, are limited to a single microstrip.

This paper presents an alternative method for efficient analysis of single and multiple coupled microstrips in a closed structure. The Chebyshev functions weighted with edge conditions [6], [8], [9] are used as basis functions to expand the unknown current densities on the strips. The product of a transformed basis function and a spectral-domain Green's 
function is a part of the total $E$-field, called the expansion $E$-field herein. The Fourier transform of the asymptotic part of each expansion $E$-field consists of slowly converging series. Through the Laplace transforms, rapidly converging summations to within an additive function can be obtained [5]. The transform of the remaining part can be handled by a regular fast Fourier transform (FFT) technique. To accelerate the computation, however, we adopt the nonuniform fast Fourier transform (NUFFT) technique [11], [12] since the sampling points are allowed to be arbitrarily located in both the original and transform domains.

After each expansion $E$-field is transformed to the spatial domain, the Galerkin's procedure is performed to set up the final MoM matrix. The integrand of each MoM entry consists of a product of an expansion $E$-field and a basis function. If the expansion $E$-fields are sampled at the nodes or abscissas of the Gauss-Chebyshev quadrature of the Lobatto's integration formulas [13], the Galerkin's integrals can be obtained without any effort by using the orthogonality of Chebyshev functions. The abscissas of the numerical quadrature are nonuniformly distributed on conducting strips, and the strips can be arbitrarily located; therefore, the NUFFT is more suitable than the regular FFT in our approach.

In the traditional SDA, the number of operations is proportional to the square of the product of the number of microstrips and the total number of basis functions for a strip. It can be anticipated that the number of operations required in the proposed method is directly proportional to the number of lines. The reason is that each of the spatial expansion $E$-fields on all the conducting strips can be obtained simultaneously after just one time the NUFFT is performed if all strips are at the same interface. This property makes the proposed method particularly suitable for analyzing a shielded microstrip structure with a large number of conducting strips.

The proposed method is also employed to investigate the propagation characteristics of MCMLs in a dual-level structure and coupled lines with finite metallization thickness. The computation requires more NUFFTs so that the relative efficiency is reduced. This paper is organized as follows. Section II addresses the NUFFT algorithm and Section III formulates the asymptotic extraction technique in the SDA. Section IV details the incorporation of the NUFFT in our approach. Section V presents the calculation results and compares the CPU time used by the proposed method and traditional SDA. Section VI draws the conclusion.

\section{NUFFT ALGORITHM}

The FFT is a fast algorithm for calculating discrete Fourier transforms, and has widespread applications in communication engineering and applied mathematics. It requires that the sampled data should be equally spaced. Recently, a generalizing FFT algorithm [11] is presented to deal with nonuniformly or unequally spaced data. Later, a new algorithm (called the NUFFT [12]) with improved accuracy is proposed without increasing the computation complexity. The idea of the NUFFT is to approximate a nonuniform sample point in the space domain by interpolating an oversampled uniform Fourier basis using a FFT with finite nonzero coefficients.

Consider the following summation with unequally spaced output data $d_{j}$ :

$$
d_{j}=\sum_{k=-N_{f} / 2}^{N_{f} / 2-1} f_{k} e^{i k s_{j}}, \quad j=1,2, \ldots, N_{d}
$$

where the sampled nodes $s_{j} \in[-\pi, \pi]$ and $s_{j}$ 's can be unequally spaced. The input sequence $\left\{f_{k}\right\}$ can be equally or unequally spaced, and $N_{f}$ and $N_{d}$ are the numbers of input and output data points, respectively. It is noted that $N_{d}=N_{f}$ is used in [11] and [12]; however, it is found in this study that $N_{d}$ can be different from $N_{f}$. This property greatly extends the flexibility of the NUFFT algorithm since the numbers of the input and output data points can be adjusted according to the requirement in the application.

To evaluate (1) with unequally spaced $s_{j}$, the key step of the NUFFT is to approximate each $e^{i k s_{j}}$ with a sum of weighted complex exponentials at $q+1$ equally spaced nodes in the neighborhood of $s_{j}$ as follows:

$$
e^{i k s_{j}} \cong \varphi_{k}^{-1} \sum_{\ell=1}^{q+1} \gamma_{\ell}\left(s_{j}\right) e^{i\left(v_{j}+\ell-q / 2-1\right) 2 \pi k / m N_{f}}
$$

where $q$ is an even positive integer and $m \geq 2$ is an index indicating the oversampling rate of the approximation. The sampling points of the complex exponentials on the right-hand side of (2) collocate with those of a regular FFT with size $m N_{f} \geq$ $2 N_{f}$. The larger the value of $m$ is, the more accurate the results are, but the larger size of the regular FFT is required. However, in all our case studies, $m=2$ and $q=8$ are sufficient to obtain a good approximation. In (2), $v_{j}=\left[s_{j} m N_{f} / 2 \pi\right]$ denotes the integer nearest to $s_{j} m N_{f} / 2 \pi$. The $q+1$ interpolation coefficients for each $s_{j}, \gamma_{1}\left(s_{j}\right), 1 \leq \ell \leq q+1$ are given as [12]

$$
\gamma=\mathbf{F}^{-1} \mathbf{P}
$$

where the entries of the $(q+1) \times(q+1)$ matrix $\mathbf{F}$ and $(q+1) \times 1$ column vector $\mathbf{P}$ are

$$
\begin{aligned}
& F_{\ell n} \\
& =\left\{\begin{array}{l}
\frac{e^{i(\ell-n) / m \pi}-e^{-i \ell-n / m \pi}}{1-e^{-i 2 \ell-n \pi / m N_{f}}}, \quad \ell \neq n \\
N_{f}, \quad \ell=n
\end{array}\right. \\
& P_{\ell}\left(s_{j}\right) \\
& =i \frac{\sin \left(\frac{2 \ell-q-2 \delta_{j}-3}{2 m} \pi\right)}{1-e^{-i\left(2 \ell-q-2 \delta_{j}-3\right) / m N_{f} \pi}}+i \frac{\sin \left(\frac{2 \ell-q-2 \delta_{j}-1}{1-e^{-i\left(2 \ell-q-2 \delta_{j}-1\right) / m N_{f} \pi}}\right)}{14)}
\end{aligned}
$$

and

$$
\delta_{j}=\frac{s_{j} m N_{f}}{2 \pi}-v_{j}
$$

The accuracy factors $\varphi_{k}$ 's in (2) are chosen to minimize the error of approximation in the least square sense. The existence of the closed-form expression of (5) relies on the choice of $\varphi_{k}=$ $\cos \left(\pi k / m N_{f}\right)$ [12]. The values of $\gamma_{\ell}\left(s_{j}\right)$ can be determined and 


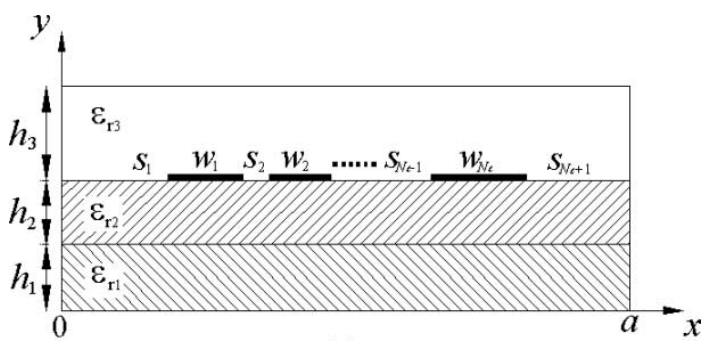

(a)

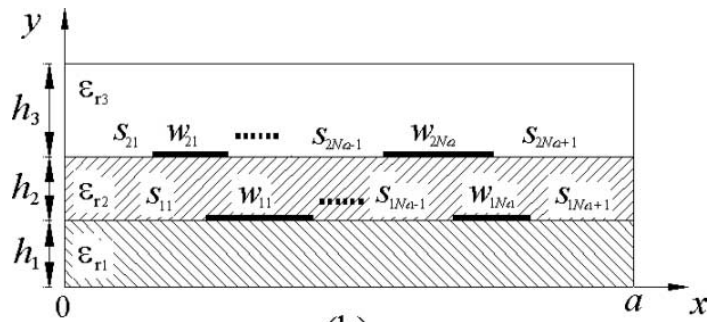

(b)

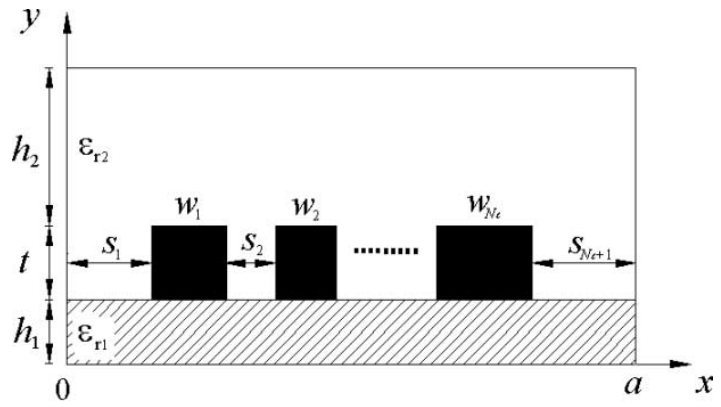

(c)

Fig. 1. Three analyzed microstrip structures. (a) All MCMLs at one interface of the dielectric layers. (b) MCMLs at two interfaces of the dielectric layers. (c) MCMLs with finite metallization thickness.

stored in memory before the microstrip structure is simulated when $s_{j}$ and $m N_{f}$ are specified. Substituting (2) into (1) yields

$$
\begin{aligned}
d_{j} & =\sum_{\ell=1}^{q+1} \gamma_{\ell}\left(s_{j}\right)\left[\sum_{k=-N_{f} / 2}^{N_{f} / 2-1}\left(\varphi_{k}^{-1} f_{k}\right) e^{i\left(v_{j}+\ell-q / 2-1\right) 2 \pi k / m N_{f}}\right] \\
& =\sum_{\ell=1}^{q+1} \gamma_{\ell}\left(s_{j}\right) Q_{\ell}\left(v_{j}\right) .
\end{aligned}
$$

A regular FFT of size $m N_{f}$ can now be invoked to evaluate $Q_{\ell}\left(-m N_{f} / 2+j\right), j=0,1, \ldots, m N_{f}-1$. In (7), only $q+1$ entries of $Q_{\ell}$ are required in the summation for each $s_{j}$. It has been shown in [12] that the accuracy of the NUFFT algorithm is much better than previously reported results in [11] with an identical computation complexity of $O\left(m N_{f} \log _{2} m N_{f}\right)$.

\section{APPROACH}

In the SDA formulation for the shielded MCMLs in Fig. 1(a) and (b), the Green's functions [14] can be written as a sum of asymptotic and remaining parts, e.g.,

$$
\tilde{G}_{z z}(\beta, n)=\tilde{G}_{z z}^{\infty}(\beta, n)+\tilde{G}_{z z}^{\mathrm{re}}(\beta, n) .
$$

where $\beta$ is the modal propagation constant to be determined, and $\tilde{G}_{z z}^{\infty}(\beta, n)$ is $\tilde{G}_{z z}(\beta, n)$ in the limit of $n \rightarrow \infty$. For a complex mode, the propagation constant is denoted as $\beta-j \alpha$. It can be shown that $\tilde{G}_{i j}^{\infty}(\beta, n), i, j=z$, or $x$ can be written as $C_{i j}(\beta)$. $\tilde{F}_{i j}(n)$ with

$$
\tilde{F}_{z z}(n)=\left|\alpha_{n}\right|^{-1} \quad \tilde{F}_{z x}(n)= \pm \alpha_{n}^{0} \quad \tilde{F}_{x x}(n)=\left|\alpha_{n}\right|
$$

if the observation fields and the currents are at the same interface, and

$$
\begin{aligned}
& \tilde{F}_{z z}(n) \propto e^{-\alpha_{n} h_{2}}\left|\alpha_{n}\right|^{-1} \\
& \tilde{F}_{z x}(n) \propto \pm e^{-\alpha_{n} h_{2}} \alpha_{n}^{0} \\
& \tilde{F}_{x x}(n) \propto e^{-\alpha_{n} h_{2}}\left|\alpha_{n}\right|
\end{aligned}
$$

if they are at different interfaces. $C_{i j}(\beta)$ is either $\beta$ or $\beta^{2}$ times a material constant, and the spectral variables $\alpha_{n}=n \pi / a$.

In the solution procedure, the basis functions for the unknown current densities on the strips are [6]

$$
\begin{aligned}
J_{z}(x) & =\sum_{i=1}^{N_{\ell}} \sum_{p=0}^{N_{b}-1} a_{i p} \frac{T_{p}\left(X_{i}\right)}{\sqrt{1-X_{i}^{2}}} \\
J_{x}(x) & =\sum_{i=1}^{N_{\ell}} \sum_{p=0}^{N_{b}-1} b_{i p} \sqrt{1-X_{i}^{2}} U_{p}\left(X_{i}\right) \\
X_{i} & = \begin{cases}\frac{2\left(x-x_{i}\right)}{w_{i}}, \quad x_{i}-\frac{w_{i}}{2} \leq x \leq x_{i}+\frac{w_{i}}{2} \\
0, \quad \text { otherwise. }\end{cases}
\end{aligned}
$$

where $x_{i}$ and $w_{i}$ are the center and width of strip $i$, respectively, $N_{\ell}$ is the number of strips, and $N_{b}$ is the number of basis functions for expanding $J_{z}$ or $J_{x}$ on a strip. $T_{j}(x)$ and $U_{j}(x)$ stand for the Chebyshev polynomials of the first and second kinds, respectively. It has been shown that the transformed basis functions are the Bessel functions of the first kind [2], [6]. In the spectral domain, a product of a transformed basis function and a Green's function is an expansion $E$-field tangential to the plane at $y=h_{1}$ or $h_{1}+h_{2}$. When these $E$-fields are transformed into the spatial domain, as indicated in (8), they can be divided into an asymptotic part, denoted with superscript $\infty$ and a remaining part, with superscript re as

$$
\left[\begin{array}{l}
E_{z}(\beta, x) \\
E_{x}(\beta, x)
\end{array}\right]=\left[\begin{array}{l}
E_{z}^{\infty}(\beta, x) \\
E_{x}^{\infty}(\beta, x)
\end{array}\right]+\left[\begin{array}{c}
E_{z}^{\mathrm{re}}(\beta, x) \\
E_{x}^{\mathrm{re}}(\beta, x)
\end{array}\right]
$$

where

$$
\begin{aligned}
& {\left[\begin{array}{l}
E_{z}^{\infty}(\beta, x) \\
E_{x}^{\infty}(\beta, x)
\end{array}\right]} \\
& \quad=\sum_{i} \sum_{p}\left[\begin{array}{c}
a_{i p} C_{z z}(\beta) E_{z z i p}^{\infty}(x)+b_{i p} C_{z x}(\beta) E_{z x i p}^{\infty}(x) \\
a_{i p} C_{x z}(\beta) E_{x z i p}^{\infty}(x)+b_{i p} C_{x x}(\beta) E_{x x i p}^{\infty}(x)
\end{array}\right]
\end{aligned}
$$

and

$$
\begin{aligned}
& {\left[\begin{array}{l}
E_{z z}^{\mathrm{re}}(\beta, x) \\
E_{x}^{\mathrm{re}}(\beta, x)
\end{array}\right]} \\
& \quad=\sum_{n=-\infty}^{\infty}\left[\begin{array}{cc}
\tilde{G}_{z z}^{\mathrm{re}}(\beta, n) & \tilde{G}_{z x}^{\mathrm{re}}(\beta, n) \\
\tilde{G}_{x z}^{\mathrm{re}}(\beta, n) & \tilde{G}_{x x}^{\mathrm{re}}(\beta, n)
\end{array}\right]\left[\begin{array}{c}
\tilde{J}_{z z}(n) \\
\tilde{J}_{x}(n)
\end{array}\right] e^{i \alpha_{n} x} .
\end{aligned}
$$

In (12c), $\tilde{J}_{t}(n), t=x$, or $z$ represents the Fourier transform of the total current distribution on a strip. In (12b), $E_{u t i p}^{\infty}(x)$, $u, t=x$, or $z$ is the inverse Fourier transform (IFT) of the 
product of $\tilde{F}_{u t}(n)$ in (10) and $p$ th basis function of $\tilde{J}_{t}(n)$ for the strip $i$. When the observation fields and currents are at the same interface, $E_{x t}^{\infty}(x)$ and $E_{z t}^{\infty}(x)$ can be expressed as

$$
\begin{aligned}
& E_{x t i p}^{\infty}(x)=\sum_{n} B_{p}\left(\frac{\alpha_{n} w_{i}}{2}\right) e^{j \alpha_{n}\left(x-x_{i}\right)} \\
& E_{z \text { tip }}^{\infty}(x)=\sum_{n} \frac{B_{p}\left(\frac{\alpha_{n} w_{i}}{2}\right)}{\alpha_{n}} e^{j \alpha_{n}\left(x-x_{i}\right)}
\end{aligned}
$$

where $B_{p}$ is the Bessel function of order $p$ of the first kind. Evaluation of (13) may take much time since the two series converge slowly. Fortunately, based on the formulation in [5], a much more rapidly converging summation to within an additive function independent of $n$ can be obtained, and only a couple of terms are required for results with sufficient accuracy. When the observation fields and currents are at different interfaces, $E_{x t}^{\infty}(x)$ and $E_{z t}^{\infty}(x)$ can be quickly obtained by numerical calculations since they are exponentially decay functions. The NUFFT processing of the remaining parts will be addressed in Section IV.

The unknown coefficients $a_{i p}$ 's and $b_{i p}$ 's in (11) can be resolved by employing the Galerkin's procedure in the spatial domain, i.e.,

$$
\begin{array}{r}
\int_{\text {strip } i} E_{z}(\beta, x) \frac{T_{p}\left(X_{i}\right)}{\sqrt{1-X_{i}^{2}}} d x=0 \\
\int_{\text {strip } i} E_{x}(\beta, x) \sqrt{1-X_{i}^{2}} U_{p}\left(X_{i}\right) d x=0
\end{array}
$$

for $i=1, \ldots, N_{\ell}$, and $p=0,1, \ldots, N_{b}-1$, and a matrix of $2 N_{\ell} N_{b} \times 2 N_{\ell} N_{b}$ can be established. By enforcing the determinant of this matrix to be zero, the propagation constant $\beta$ for any mode of the MCMLs can be solved, and the entries of the corresponding eigenvector are the unknown coefficients.

\section{INCORPORATING THE NUFFT INTO THE ANALYSIS}

The NUFFT algorithm is employed to calculate these summations in (12c), which will converge if $n$ is truncated at a sufficiently large number $N_{f}$. The total $E$-fields in (12a) can be expressed as

$$
\begin{aligned}
& E_{z}(x)=\sum_{i=1}^{N_{\ell}} \sum_{p=0}^{N_{b}-1} a_{i p} E_{z z i p}(x)+\sum_{i=1}^{N_{\ell}} \sum_{p=0}^{N_{b}-1} b_{i p} E_{z x i p}(x) \\
& E_{x}(x)=\sum_{i=1}^{N_{\ell}} \sum_{p=0}^{N_{b}-1} a_{i p} E_{x z i p}(x)+\sum_{i=1}^{N_{\ell}} \sum_{p=0}^{N_{b}-1} b_{i p} E_{x x i p}(x)
\end{aligned}
$$

where the expansion $E$-field, say, $E_{z x i p}(x)$, is a partial longitudinal component resulted from the IFT of the product of $\tilde{G}_{z x}$ and the transform of the $p$ th basis function of $J_{x}$ for strip $i$ in (11b). Let the expansion $E$-fields be expanded in a convergent series as

$$
E_{z t i p}(x)=\sum_{q=0}^{N_{g}-1} d_{j q} T_{q}\left(X_{j}\right)
$$

or

$$
E_{x t i p}(x)=\sum_{q=0}^{N_{g}-1} h_{j q} U_{q}\left(X_{j}\right)
$$

where $t=x$ or $z$, and $N_{g}$ is the number of Chebyshev functions for the approximation. It is obvious that the results of (14) will simply be $d_{j q}$ or $h_{j q}$ weighted by proper constants [13] by utilizing the orthogonal property of the Chebyshev polynomials. For example, the coefficients $d_{j q}$ are given by

$$
\begin{aligned}
d_{j q} & =\frac{2}{\pi} \int_{-1}^{1} \frac{E_{z t i p}(x) T_{q}\left(X_{j}\right)}{\sqrt{1-X_{j}^{2}}} d X_{j} \\
& \approx \frac{2}{N_{g}} \sum_{k=0}^{N_{g}-1} E_{z t i p}\left(x_{j k}\right) \cos q \theta_{k} \\
\theta_{k} & =\frac{2 k+1}{2 N_{g}} \pi, \quad k=0,1, \ldots, N_{g}-1 .
\end{aligned}
$$

It means that if the sample points are $x_{j k}=x_{j}+\left(w_{j} / 2\right) \cos \theta_{k}$, i.e., the nodes of a Gauss-Chebyshev quadrature [13], the evaluation of (14) will require no computation effort. It is noted that $N_{g}$ in (17) also represents the number of nodes of the numerical quadrature so it must be no less than $N_{b}$. A database for the sampling nodes can be established before the program is executed. A general structure of MCMLs can have any number of lines with arbitrary widths, spacings, and positions. Consequently, it would be impossible to have the sampled points for the $E$-fields be uniformly distributed on the conducting strips. This is the very reason why the NUFFT algorithm is particularly suitable for this approach.

In the traditional SDA, the number of operations for establishing the final MoM matrix is proportional to $N_{s}\left(N_{\ell} N_{b}\right)^{2}$, with $N_{s}$ being the number of spectral terms in the summation. It can be shown that the number of operations for the proposed method is $2 N_{\ell} N_{b}\left[\left(m N_{f}\right) \log _{2}\left(m N_{f}\right)\right]$, excluding the operations for a Gauss-Chebyshev quadrature. The term $\left(m N_{f}\right) \log _{2}\left(m N_{f}\right)$ is the number of operations for a regular FFT of size $m N_{f}$. Thus, the proposed method is particularly suitable for analyzing MCMLs with a large number of strips at one dielectric interface.

For microstrips with finite metallization thickness, as shown in Fig. 1(c), the mixed spectral-domain approach (MSDA) [7] can be invoked. The unknown variables become the tangential $E$-fields (or equivalent magnetic current sources) in apertures. The MSDA Green's functions have similar asymptotic properties to those in (9) and (10), and the approach in (12)-(17) are still applicable to this case if the basis functions in (11) are adopted. It is worth mentioning that extra NUFFTs have to be performed to obtain the spatial expansion $H$-fields in the apertures in Fig. 1(c) at $y=h_{1}^{+}$and $h_{1}+t^{-}$. Thus, the improvement in computation using this approach will be degraded.

\section{RESUlTS AND DISCUSSIONS}

Here, convergence tests are performed and the accuracy of the results is examined before the propagation characteristics of microstrips are presented. The used CPU time of the proposed method is then compared with that of the conventional SDA and Ansoft HFSS, version 7. In the NUFFT approximation (2), $m=2$ and $q=8$ are used. 
TABLE I

CONVERGENCE ANALYSIS AND COMPARISON OF THE CPU TIME FOR A QUASI-TEM MODE OF AN EIGHT-LINE MicROSTRIP STRUCTURE OBTAINED BY THE TRADITIONAL SDA AND THE PROPOSED METHOD

\begin{tabular}{|c|c|c|c|c|c|c|}
\hline \multicolumn{2}{|c|}{ Traditional SDA } & \multicolumn{5}{|c|}{$\beta / k_{0}$ (CPU time in seconds) by the NUFFT } \\
\hline$N_{s}$ & $\beta / k_{0}$ (CPU) & $N_{b}$ & $N_{\mathrm{g}}$ & $N_{f}=64$ & $N_{f}=128$ & $N_{f}=1024$ \\
\hline $1 \times 10^{3}$ & $2.63324(3.30)$ & & 2 & $2.60926(0.50)$ & $2.60941(0.57)$ & $2.60944(1.59)$ \\
\hline $10^{3}$ & $2.61352(6.46)$ & & 64 & $2.60925(1.93)$ & $2.60940(1.99)$ & $2.60943(3.07)$ \\
\hline $5 \times 10^{3}$ & $2.61011(18.5)$ & & 3 & $2.60912(0.80)$ & $2.60926(0.91)$ & $2.60929(2.42)$ \\
\hline $1 \times 10^{4}$ & $2.60963(38.8)$ & & 64 & $2.60913(2.95)$ & $2.60927(3.04)$ & $2.60931(4.63)$ \\
\hline$\times 10^{4}$ & $2.60945(78.3)$ & & 4 & $2.60913(1.15)$ & $2.60927(1.28)$ & $2.60930(3.27)$ \\
\hline $5 \times 10^{4}$ & $2.60935(192)$ & & 64 & $2.60913(4.00)$ & $2.60927(4.11)$ & $2.60931(6.18)$ \\
\hline $1 \times 10^{5}$ & $2.60933(384)$ & & 5 & $2.60913(1.53)$ & $2.60927(1.69)$ & $2.60930(4.15)$ \\
\hline $2 \times 10^{5}$ & $2.60931(767)$ & & 64 & $2.60913(5.04)$ & $2.60927(5.15)$ & $2.60931(7.74)$ \\
\hline
\end{tabular}

\section{A. Validity Check}

For an eight-line microstrip in Fig. 1(a), let the structural parameters be $\varepsilon_{r 1}=\varepsilon_{r 2}=8.2, \varepsilon_{r 3}=1, a=40, h_{1}+h_{2}=1.8$, $h_{3}=5.4, w_{1}-w_{8}$ be $0.26,0.22,0.18,0.14,0.16,0.2,0.24$ and 0.28 , and $s_{1}-s_{9}$ be $18.495,0.25,0.21,0.17,0.15,0.19,0.23$, 0.27 , and 18.355. All dimensions are in millimeters and the frequency is $10 \mathrm{GHz}$. Table I compares the convergence and the CPU time for calculating the normalized propagation constant of a propagation mode obtained by the traditional SDA and the proposed method. In the traditional SDA, $N_{b}=4$ is used, and the final MoM matrix has a size of $64 \times 64$. If we assume that the result is converged at the number of spectral terms $N_{s}=2 \times 10^{5}$, then $N_{s} \geq 5 \times 10^{3}$ will be required for a result with a relative deviation less than $0.05 \%$. In the proposed method, the convergence behavior of the results depends on the values of $N_{g}, N_{b}$, and $N_{f}$. Here, one $N_{g}$ value is used for the eight strips. First, the results remain almost unchanged as $N_{b}$ is increased from 3 to 5, for the listed $N_{f}$ and $N_{g}$ values. When $N_{g}$ is increased from $N_{b}$ to 64 , only the sixth significant decimal digit of the results changes. There are two significant facts that are not shown in this table. First, the results for $N_{g}=8,16$, and 32 are between those for $N_{g}=N_{b}$ and $N_{g}=64$. When $N_{f}$ is changed from 64 to 128 , however, the fifth significant decimal digit changes. This indicates that the number of spectral terms for evaluating $\tilde{G}^{\text {re }}$ in (8) or $E^{\text {re }}$ in (12c) can be critical for obtaining highly accurate results. Second, the results for $N_{f}=256$ and 512 are between those for $N_{f}=128$ and 1024, and those for $N_{f}=2048$ agree with those for $N_{f}=1024$ to at least six significant decimal digits.

In Table I, the numbers in the parentheses next to the normalized propagation constants are the CPU time for calculating the results. The CPU time is measured with a MATLAB program, version 5.3 on a PC with a Pentium III processor of $900 \mathrm{MHz}$. If the NUFFT result with $N_{b}=N_{g}=4, N_{f}=128$ is compared with the SDA result with $N_{s}=2 \times 10^{4}$, an improved computation efficiency of 60 times is obtained. We also use the Ansoft's HFSS with a Pentium III processor of $1 \mathrm{GHz}$ to simulate the structure. The result is 2.6061 and it takes $33 \mathrm{~s}$.

In Table II, results reported in [15] are referred to validate the proposed method. If Fig. 1(a) is referred, $\varepsilon_{r 1}=\varepsilon_{r 3}=1$, $\varepsilon_{r 2}=8.2, a=18, w=1.8, s_{1}=s_{2}=8.1, h_{1}=h_{2}=1.8$, and $h_{3}=5.4$, all in millimeters. In the proposed method, $N_{b}=$ $N_{g}=4$ are used. As indicated in this table, $N_{f}=128$ can be
TABLE II

VAlidity Check of the Modal SOlutions ObTained By the Proposed Method. StRUCTURE IN Fig. 1(a): $\varepsilon_{r 1}=\varepsilon_{r 3}=1, \varepsilon_{r 2}=8.2$, $a=18, w=1.8, s_{1}=s_{2}=8.1, h_{1}=h_{2}=1.8$, AND $h_{3}=5.4$, ALL IN MILLIMETERS

\begin{tabular}{c|c||c|c|c|c|c}
\hline \multicolumn{2}{c||}{$[15]$} & \multicolumn{5}{c}{ This method } \\
\hline$f(\mathrm{GHz})$ & $(\beta-j \alpha) / k_{0}$ & $N_{f}=16$ & $N_{f}=32$ & $N_{f}=64$ & $N_{f}=128$ & $N_{f}=1024$ \\
\hline \multirow{4}{*}{11.63} & 1.9817 & 1.98153 & 1.98170 & 1.98171 & 1.98172 & 1.98172 \\
\cline { 2 - 7 } & 0.9024 & 0.90242 & 0.90242 & 0.90242 & 0.90242 & 0.90242 \\
& -0.0001 & $-j 0.00015$ & $-j 0.00014$ & $-j 0.00014$ & $-j 0.00014$ & $-j 0.00014$ \\
\cline { 2 - 7 } & 0.3589 & 0.35894 & 0.35894 & 0.35894 & 0.35894 & 0.35894 \\
& $-j 0.0881$ & $-j 0.08912$ & $-j 0.08851$ & $-j 0.08850$ & $-j 0.08844$ & $-j 0.08844$ \\
\hline \multirow{4}{*}{12.70} & 2.0241 & 2.02384 & 2.02405 & 2.02406 & 2.02407 & 2.02407 \\
\cline { 2 - 7 } & 1.1286 & 1.12850 & 1.12854 & 1.12855 & 1.12855 & 1.12855 \\
\cline { 2 - 7 } & 0.8633 & 0.86329 & 0.86333 & 0.86334 & 0.86334 & 0.86334 \\
\cline { 2 - 7 } & 0.2686 & 0.26854 & 0.26859 & 0.26859 & 0.26859 & 0.26859 \\
\hline
\end{tabular}

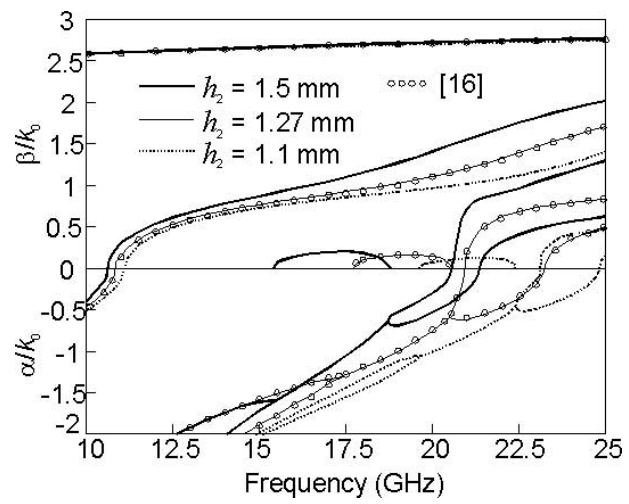

Fig. 2. Dependence of modal propagation constants for a shielded single microstrip on the thickness of a dielectric substrate. Structural parameters are $a=12.7 \mathrm{~mm}, w=1.27 \mathrm{~mm}, h_{1}=0 \mathrm{~mm}, h_{3}=11.43 \mathrm{~mm}$, $s_{1}=s_{2}=(a-w) / 2$, and $\varepsilon_{r}=8.875$.

used for obtaining results for various modes converged to three decimal significant digits. The results are in close agreement with the referred data.

\section{B. Modal Propagation Characteristics for Single and Multiple Coupled Microstrips}

Fig. 2 shows the charts for some leading modes of a shielded single microstrip line on a substrate with $\varepsilon_{r}=8.875$ and thickness $h_{2}=1.1,1.27$, and $1.5 \mathrm{~mm}$. The modal solutions, including quasi-TEM, higher order, evanescent, and complex modes, with $h_{2}=1.27 \mathrm{~mm}$ have a good agreement with those in [16]. As shown in Fig. 2, the propagation constants for the quasi-TEM modes do not vary significantly as $h_{2}$ is changed. For the first higher order modes, however, the mode charts have significantly deviations when frequency is higher than $20 \mathrm{GHz}$. The frequency band with complex modes migrates to higher frequencies when the thickness of the substrate is decreased.

Fig. 3 shows the dispersive propagation characteristics of the quasi-TEM modes of an eight-line microstrip structure, of which the structural parameters are identical to those in Table I. The normalized phase constants for the two leading modes are referred to the vertical axis on the right, while those of the others are referred to that on the left. At the lower frequency end of interest, the fourth through the eighth modes have very close phase constants. The program has to solve these 


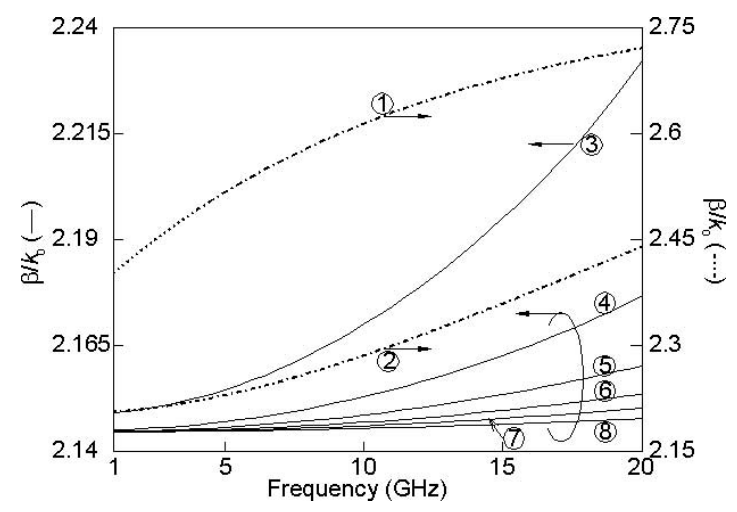

Fig. 3. Dispersive normalized phase constants for the quasi-TEM modes of a shielded eight-line microstrip structure. Structural parameters are identical to those of Table I.

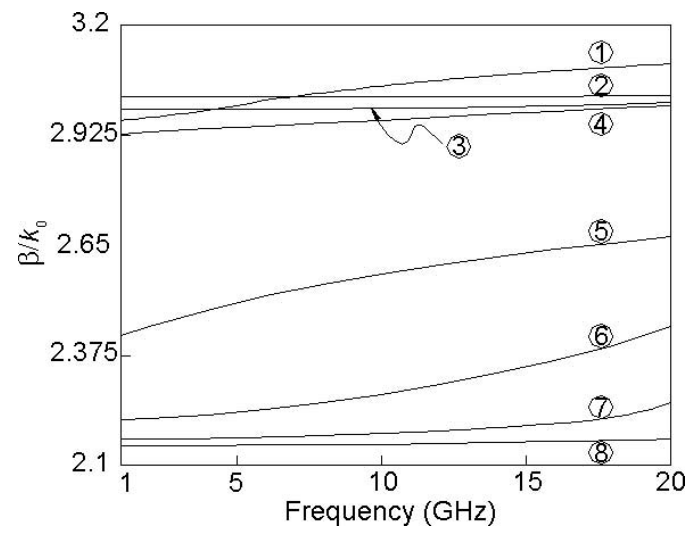

Fig. 4. Dispersive normalized phase constants for a dual-level shielded eight-line microstrip structure. Structure parameters are $\varepsilon_{r 1}=10.2$, $\varepsilon_{r 2}=8.2, \varepsilon_{r 3}=1, a=40, h_{1}=1.27, h_{2}=0.53, h_{3}=5.4, w_{11}-w_{14}$ are $0.22,0.14,0.2$, and $0.28, w_{21}-w_{24}$ are $0.26,0.18,0.16$, and $0.24, s_{11}-s_{15}$ are $19.005,0.56,0.5,0.74$, and 18.355 , and $s_{21}-s_{25}$ are $18.495,0.68,0.46$, 0.62 , and 18.905. All dimensions are in millimeters.

normalized modes with an accuracy of at least four significant decimal digits to distinguish their values.

It is worth mentioning that it will be difficult using the finite-element HFSS to generate the whole mode charts shown in Figs. 2 and 3. The reason is as follows. In using HFSS for calculating the propagation constant of a transmission line, one has to key in a guessed phase difference for the period in the longitudinal direction of the periodic structure for simulation. This approach works well for normal modes. When evanescent or complex modes are required, however, the total phase difference of the simulation circuit becomes problematic. For the results with a high resolution, HFSS will need many more passes to assure and justify the converged solutions than that for a single mode.

Fig. 4 plots the normalized phase constants of the quasi-TEM modes for a shielded dual-level eight-line microstrip structure. The structure is changed from that in Fig. 3, leaving strips 1, 3, 5 , and 7 on the top level and moving strips $2,4,6$, and 8 to the interface between layers 1 and 2. The CPU time for obtaining the eight modes at one frequency point by the proposed method with $N_{b}=N_{g}=4$ and $N_{f}=128$ is $3.03 \mathrm{~s}$, and that by the traditional SDA with $N_{b}=4$ and $N_{s}=2 \times 10^{4}$ is $70.21 \mathrm{~s}$. The improved computation efficiency is reduced to 23 times.

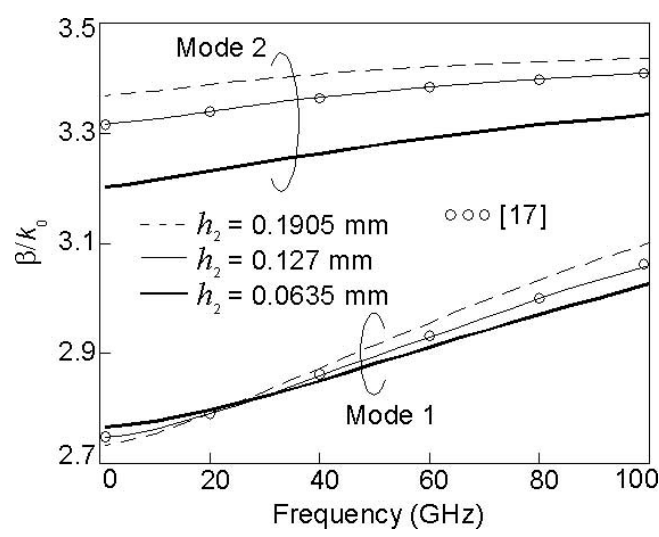

Fig. 5. Dispersive normalized phase constants for a dual-level microstrip structure. Structural parameters in Fig. 1(b) are $a=25.4 \mathrm{~mm}$, $h_{1}=w_{11}=w_{21}=0.127 \mathrm{~mm}, h_{3}=25.146 \mathrm{~mm}, s_{11}=s_{22}=12.895 \mathrm{~mm}$, $s_{12}=s_{21}=12.378 \mathrm{~mm}, \varepsilon_{r 1}=\varepsilon_{r 2}=12$, and $\varepsilon_{r 3}=1$.

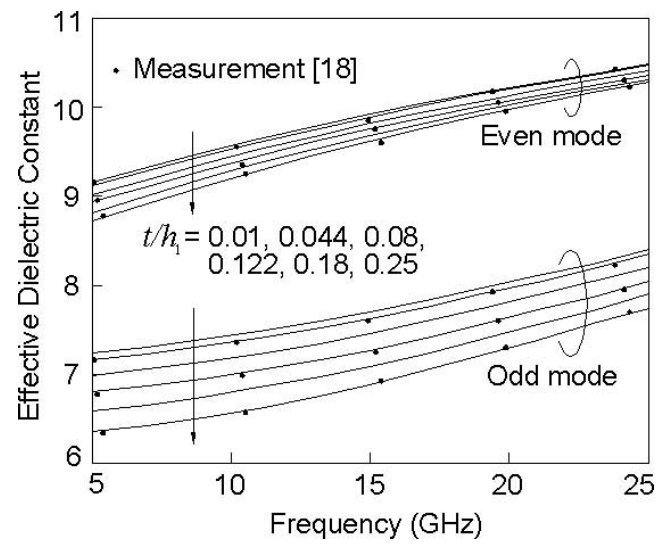

Fig. 6. Effective constants for a pair of coupled microstrip lines with finite metallization thickness. Structural parameters in Fig. 1(c) are $\varepsilon_{r 1}=12.5$, $\varepsilon_{r 2}=1, w_{1}=w_{2}=s_{2}, h_{1}=0.6 \mathrm{~mm}, h_{2}=10 \mathrm{~mm}$, and $s_{1}=s_{3}=6 \mathrm{~mm}$.

Fig. 5 shows the dispersive $\beta / k_{o}$ for dual-level coupled lines in Fig. 1(b) with $a=25.4 \mathrm{~mm}, h_{1}=w_{11}=w_{21}=0.127 \mathrm{~mm}$, $h_{3}=25.146 \mathrm{~mm}, s_{11}=s_{22}=12.895 \mathrm{~mm}, s_{12}=s_{21}=$ $12.378 \mathrm{~mm}, \varepsilon_{r 1}=\varepsilon_{r 2}=12$, and $\varepsilon_{r 3}=1$. The three plots have $h_{2}=0.0635 \mathrm{~mm}, 0.127 \mathrm{~mm}$, and $0.1905 \mathrm{~mm}$. The results with $h_{2}=0.127 \mathrm{~mm}$ have a good agreement with those in [17].

Fig. 6 plots the dispersive characteristics of a pair of coupled microstrips in Fig. 1(c) for various values of $t$, the metallization thickness. The measured results are referred to [18]. We choose the case with $t / h_{1}=0.01$ at $5 \mathrm{GHz}$ for comparing the used CPU time by the proposed method with that by the MSDA. This small thickness may introduce a serious convergence problem to the MSDA program since a large number of spectral summation terms are required to obtain accurately converged data. It is found that the MSDA provides $\beta / k_{o}=$ $3.0379,3.0381$ and 3.0382 when $N_{b}=4,5$ and 6 , respectively, at $N_{s}=2 \times 10^{5}$. Thus, it is reasonable to assume the converged result to be 3.0382 . Table III lists the $\beta / k_{o}$ values and the CPU time used by the MSDA and the proposed method. If an agreement to five significant decimal digits is required, the CPU time required for proposed method is between 16-20 s, while that for the MSDA is $75 \mathrm{~s}$. The CPU time ratio is reduced approximately four times. The reduction is obviously resulted 
TABLE III

CONVERGENCE ANALYSIS AND COMPARISON OF THE CPU TIME FOR AN ODD MODE OF A PAIR OF COUPLED LiNES WITH $t / h_{1}=0.01$ OBTAINED BY THE MSDA AND THE PROPOSED METHOD

\begin{tabular}{|c|c|c|c|c|c|c|}
\hline \multicolumn{2}{|r|}{ ISDA } & \multicolumn{5}{|c|}{$\beta / k_{0}$ (CPU time in seconds) by the NUFFT } \\
\hline$N_{s}$ & $\beta / k_{0}($ & $N_{b}$ & $N_{g}$ & $N=$ & $N k_{k}=$ & 24 \\
\hline & 37 & & 32 & $382(10.06)$ & 1.83) & 3.038 \\
\hline & 3.0381 & & 64 & & & \\
\hline $10^{4}$ & 3.0382 & & 32 & 3.038 & 54) & 3.03 \\
\hline $10^{4}$ & 3.0382 & & 64 & 3.0383 & 3.0382 & |3.038 \\
\hline $10^{5}$ & 3.038 & & 32 & 3.03 & 3.038 & 3.03 \\
\hline & & & 64 & & & 03 \\
\hline
\end{tabular}

from the fact that the unknown variables are now at four dielectric interfaces. Moreover, with reference to Fig. 1(c), there are three "isolated" apertures at $y=h_{1}^{+}$and $y=h_{1}+t^{-}$so extra times of the NUFFT will be invoked, and this causes the proposed method to increase the CPU time significantly.

\section{CONCLUSION}

The NUFFT algorithm and asymptotic extraction technique have been combined to efficiently and accurately analyze the propagation characteristics of single and multiple coupled microstrips in a shielded enclosure. The computation is further accelerated by a Gauss-Chebyshev quadrature. For an infinitely thin eight-line microstrip structure, the CPU time used by the proposed method is only one 60th of that by the traditional SDA for results with similar accuracy. When the method is applied to microstrips at different dielectric interfaces or with finite metallization thickness, the computational efficiency is reduced from four to ten.

\section{REFERENCES}

[1] F. Liu, J. E. Schutt-Ainé, and J. Chen, "Full-wave analysis and modeling of multiconductor transmission lines via 2-D-FDTD and signalprocessing techniques," IEEE Trans. Microwave Theory Tech., vol. 50, pp. 570-577, Feb. 2002.

[2] J.-T. Kuo and C.-K. C. Tzuang, "Complex modes in shielded suspended coupled microstrip lines," IEEE Trans. Microwave Theory Tech., vol. 38, pp. 1278-1286, Sept. 1990

[3] J. Bernal, F. Medina, R. Boix, and M. Horno, "Fast full-wave analysis of multistrip transmission lines based on MPIE and complex image theory," IEEE Trans. Microwave Theory Tech., vol. 48, pp. 445-452, Mar. 2000.

[4] C. J. Railton and T. Rozzi, "Complex modes in boxed microstrip," IEEE Trans. Microwave Theory Tech., vol. 36, pp. 865-874, May 1988.

[5] C. J. Railton and J. P. McGeehan, "A rigorous and computationally efficient analysis of microstrip for use as an electro-optic modulator," IEEE Trans. Microwave Theory Tech., vol. 37, pp. 1099-1104, July 1989.

[6] V. K. Tripathi and H. Lee, "Spectral-domain computation of characteristic impedances and multiport parameters of multiple coupled microstrip lines," IEEE Trans. Microwave Theory Tech., vol. 37, pp. 215-221, Jan. 1989.

[7] J.-T. Kuo and T. Itoh, "Hybrid-mode computation of propagation and attenuation characteristics of parallel coupled microstrips with finite metallization thickness," IEEE Trans. Microwave Theory Tech., vol. 38, pp. 1278-1286, Sept. 1997.
[8] S. Park and C. A. Balanis, "Dispersion characteristics of open microstrip lines using closed-form asymptotic extraction," IEEE Trans. Microwave Theory Tech., vol. 45, pp. 458-460, Mar. 1997.

[9] —-, "Closed-form asymptotic extraction method for coupled microstrip lines," IEEE Microwave Guided Wave Lett., vol. 7, pp. 84-86, Mar. 1997.

[10] J. L. Tsalamengas and G. Fikioris, "Rapidly converging spectral-domain analysis of rectangularly shielded layered microstrip lines," IEEE Trans. Microwave Theory Tech., vol. 51, pp. 1729-1734, June 2003.

[11] A. Dutt and V. Rokhlin, "Fast Fourier transforms for nonequispaced data," SIAM J. Sci. Comput., vol. 14, pp. 1368-1393, Nov. 1993.

[12] Q. H. Liu and N. Nguyen, "An accurate algorithm for nonuniform fast Fourier transforms (NUFFTs)," IEEE Microwave Guided Wave Lett., vol. 8, pp. 18-20, Jan. 1998.

[13] M. Abramowitz and I. A. Stegun, Eds., Handbook of Mathematical Functions With Formulas, Graphs, and Mathematical Tables. Boulder, CO: Nat. Bureau Stand., 1972, vol. Appl. Math. 55.

[14] T. Itoh, Numerical Techniques for Microwave and Millimeter-Wave Passive Structures. New York: Wiley, 1989, ch. 5.

[15] M. J. Freire, F. Mesa, and M. Horno, "Excitation of complex and backward mode on shielded lossless printed lines," IEEE Trans. Microwave Theory Tech., vol. 47, pp. 1098-1105, July 1999.

[16] W.-X. Huang and T. Itoh, "Complex modes in lossless shielded microstrip lines," IEEE Trans. Microwave Theory Tech., vol. 36, pp. 163-165, Jan. 1988.

[17] L. Crain and K. J. Webb, "An equivalent circuit model for terminated hybrid-mode multiconductor transmission lines," IEEE Trans. Microwave Theory Tech., vol. 37, pp. 1784-1793, Nov. 1989.

[18] R. T. Kollipara and V. K. Tripathi, "Dispersion characteristics of moderately thick microstrip lines by the spectral domain method," IEEE Microwave Guided Wave Lett., vol. 2, pp. 100-101, Mar. 1992.

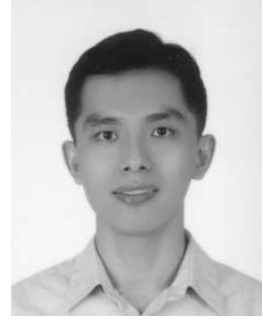

electromagnetics.
Ke-Ying Su was born in Tainan, Taiwan, R.O.C., on March 16, 1974. He received the B.S. degree in applied mathematics from the National Sun Yet-sen University (NSYSU), Taiwan, R.O.C., in 1996, the M.S. degree in mathematics from the National Central University (NCU), Taiwan, R.O.C., in 1998, and is currently working toward the Ph.D. degree in communication engineering at the National Chiao Tung University (NCTU), Taiwan, R.O.C.

His research interests include the analysis of microwave circuits and numerical techniques in

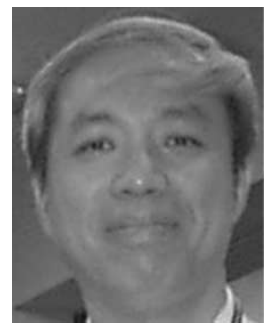

Jen-Tsai Kuo (S'88-M'92-SM'04) received the $\mathrm{Ph} . \mathrm{D}$. degree from the Institute of Electronics, National Chiao Tung University (NCTU), Hsinchu, Taiwan, R.O.C., in 1992.

Since 1984, he has been with the Department of Communication Engineering, NCTU, as a Lecturer in both the Microwave and Communication Electronics Laboratories. He is currently a Professor with the Department of Communication Engineering, and serves as the Chairman of the Degree Program of Electrical Engineering and Computer Science (EECS), NTU. During the 1995 academic year, he was a Visiting Scholar with the University of California at Los Angeles. His research interests include the analysis and design of microwave circuits, high-speed interconnects and packages, field-theoretical studies of guided waves, and numerical techniques in electromagnetics. 